\title{
NONLINEAR RIEMANN-HILBERT PROBLEMS FOR GENERALIZED ANALYTIC FUNCTIONS
}

\author{
Messoud A. Efendiev, Wolfgang L. Wendland
}

Dedicated to Professor Bogdan Bojarski on the occasion of his 75 th birthday.

\begin{abstract}
In this study we deal with the nonlinear Riemann-Hilbert problem (in short (RHP)) for generalized analytic functions in multiply connected domains. Using a similarity principle for multiply connected domains (presented here for the first time), we reduce the nonlinear RHP for generalized analytic functions to a corresponding nonlinear RHP for holomorphic functions with Hölder continuous boundary data. Then the Newton-Kantorovič method combined with a continuation procedure as well as a new existence theorem for holomorphic solutions, which is based on topological degree arguments, leads to existence of at least two topologically different generalized analytic functions solving the nonlinear RHP.
\end{abstract}

Keywords: Similarity principle for multiply connected domains, nonlinear Riemann-Hilbert problems, generalized analytic functions, topological degree

\section{Introduction}

In this article we are concerned with nonlinear Riemann-Hilbert problems for generalized analytic functions in multiply connected plane domains. Let $\Omega_{m} \subset \mathbb{C}$ be a given $(m+1)$-connected bounded domain with the boundary $\partial \Omega_{m}=\Gamma=$ $\bigcup_{j=0}^{m} \Gamma_{j}$ consisting of $m+1$ separated, smooth (at least $\Gamma \in C^{2}$ ), closed Jordan curves, and where $\Gamma_{0}$ is the exterior boundary. The closed curves $\Gamma_{j}$ have 1periodic parametric representations $\Gamma_{j}: z=t_{j}(s)$ with $s \in \mathbb{R}, j=0, \ldots, m$.

The nonlinear Riemann-Hilbert boundary value problem for generalized analytic functions in $\Omega_{m}$ reads:

Find $u(x, y)$ and $v(x, y)$ in the Hölder space $C^{\beta}\left(\bar{\Omega}_{m}\right), 0<\beta<1$ such that $w:=u+i v$ satisfies the Vekua system

$$
\frac{\partial w}{\partial \bar{z}}-A w-B \bar{w}=0 \quad \text { in } \Omega_{m}
$$

and a parameter $\omega \in \mathbb{R}^{m-1}$ such that the nonlinear boundary conditions

$$
\left.F_{j}(\omega ; z, u, v)\right|_{\Gamma_{j}}=0 \quad \text { for } z=t_{j}(s) \in \Gamma_{j}, \quad j=0,1, \ldots, m
$$

2000 Mathematics Subject Classification: 30G20, 35J65, 47H11, 45 G05. 
are satisfied and

$$
\int_{\Gamma_{j}} d \arg w=0 \quad \text { for } j=0,1, \ldots, m,
$$

where $F_{j}$ are given real-valued functions on $\mathbb{R}^{m-1} \times \mathbb{C} \times \mathbb{R}^{2}$.

The differentiation $\frac{\partial}{\partial \bar{z}}=\frac{1}{2}\left(\frac{\partial}{\partial x}+i \frac{\partial}{\partial y}\right)$ in (1.1) is the weak derivative in Sobolev's sense [15, S. 24ff.]. For the given coefficient functions in (1.1) we assume $A, B \in$ $L_{q}\left(\Omega_{m}\right)$ with $q>4$.

In order to use the geometric and topological properties of the boundary conditions (1.2), we reformulate them as follows.

For any fixed values of $\omega \in \mathbb{R}^{m-1}$ and $z \in \Gamma$, the real-valued functions $F_{j}$ define by

$$
\gamma_{\omega, z, j}:=\left\{w=u+i v \in \mathbb{C}_{w} \mid F_{j}(\omega ; z, u, v)=0, \quad z \in \Gamma_{j}\right\}
$$

a family of $m+1$ curves in the complex state plane $\mathbb{C}_{w}$. Correspondingly, the problems (1.1)-(1.3) can also be formulated as follows:

Find $w \in C^{\beta}\left(\bar{\Omega}_{m}\right)$ satisfying (1.1) in $\Omega_{m}$ and (1.3), along with the parameters $\omega \in \mathbb{R}^{m-1}$ such that the geometric boundary conditions

$$
w(z) \in \gamma_{\omega, z, j} \quad \text { for } z \in \Gamma_{j}, \quad j=0,1, \ldots, m
$$

are satisfied.

The conditions on the family of these curves will be further specified after an appropriate family of nonlinear Riemann-Hilbert problems for holomorphic functions will be associated with (1.1)-(1.5). This will be obtained by employing the similarity principle in a specific form in Section 2.

In previous works we considered Cauchy-Riemann systems in plane domains with nonlinear boundary conditions (see, e.g., [9]). As for the first order systems (1.1), the main contributions were achieved in the 50's by I.N. Vekua and W. Haack and their coworkers (see the monographs [15] and [11]). Some parts of I.N. Vekua's book [15] are in particular due to Bogdan Bojarski. There one of the basic ideas, for tackling linear boundary value problems for (1.1), is the similarity principle due to Bers [2] and Vekua [16], which allows to reduce the boundary value problems (1.1) and (1.2) to corresponding ones for holomorphic functions. This approach was successfully used for both, linear as well as nonlinear boundary value problems of the form (1.1) and (1.2) in simply connected domains (see e.g. [1, 12, 18, 19] and the comprehensive monograph by Wegert [17] and references therein). Most general strongly elliptic systems of first order were considered by Bogdan Bojarski and Tadeusz Iwaniec in $[4,5]$, and reduced to a nonlinear normal form which allows the treatment of Riemann-Hilbert boundary value problems.

In all these papers, the complex function theory in simply connected domains - as the similarity principle, the parameterization of holomorphic functions on the boundary via their real parts employing the Hilbert transform leading to asso- 
ciated, in general nonlinear, singular integral equations on the boundary - played a fundamental role. Since the authors investigated the nonlinear Riemann-Hilbert problems for holomorphic functions in multiply connected domains in $[7,8,9]$ the first of the authors was asking experts, in particular Bogdan Bojarski, for similarity principles in multiply connected domains needed for studying corresponding Riemann-Hilbert problems for generalized analytic functions, but at that time for multiply connected domains an appropriate formulation of the similarity principle was not known, although the second author could provide the desired formulation for a long time (see [18]). Hence, in this paper, our analysis is based on a representation formula for generalized analytic functions together with the similarity principle for multiply connected domains, which we present here for the first time; an appropriate parameterization of holomorphic functions, a new existence theorem for holomorphic solutions of nonlinear Riemann-Hilbert problems and a new Newton-Kantorovič method for non invertible operators possessing only a right inverse of their Frechét derivative (see [8, Appendix]), which is due to the multiple connectivity of $\Omega_{m}$ for $m \geqslant 2$.

The paper is organized as follows. Section 2 is devoted to the similarity principle for multiply connected domains. In Section 3 we employ the similarity principle for the reduction of the boundary value problem (1.1) and (1.2) to nonlinear Riemann-Hilbert problems for holomorphic functions. The corresponding boundary conditions (1.5) for the holomorphic functions, however, are multiplied by functions $\varrho_{j}(s)$, which are only Hölder continuous. Therefore, we need an extension of the method by Reissig and Wegert [12] from their simply connected to our multiply connected case to find an appropriate a priori estimate for the solutions in a Slobodeckii space and a Hölder space. For the case $\varrho_{j} \equiv 1$ we apply a new topological degree theory for quasiruled Fredholm maps on quasicylindrical domains to nonlinear singular integral equations on $\Gamma$ associated with (1.1) and (1.2) and prove global solvability for holomorphic functions presented in [9]. There we lift the problem to one on the universal covering which is a perturbation of linear problems and we need (1.3). Then the linearized Frechét derivative problems have Fredholm index $1-m$, which in general are not solvable; therefore additional unknown constants are introduced and the boundary conditions are modified correspondingly (see [6]). In Section 4, the Newton-Kantorovič method is combined with an embedding continuation method generalizing an approach in [20], which, together with the a priori estimate of subsequent solutions, provides us a path of homotopy. Finally, Schauder's fixed point theorem guarantees the existence of solutions to (1.1) and (1.2).

\section{The similarity principle}

Solutions of the homogeneous equation (1.1) are also called "generalized analytic functions" since they are closely related to complex analytic functions by the socalled similarity principle which goes back to L. Bers [2] and I.N. Vekua [16] and can be formulated in the form of the following two theorems. 
Theorem 2.1 (S1). Let $w \in C^{\beta}\left(\bar{\Omega}_{m}\right)$ with $0<\beta<1$ be a generalized solution of the Vekua system

$$
\frac{\partial w}{\partial \bar{z}}=A w+B \bar{w} \quad \text { in } \Omega_{m}
$$

with coefficients $A, B \in L_{q}\left(\Omega_{m}\right)$ where $q>4$. Then there exists a function $\widetilde{w}=$ $\widetilde{u}+i \widetilde{v} \in C^{\alpha}\left(\bar{\Omega}_{m}\right)$ with any positive $\alpha \leqslant 1-2 / q$ and satisfying

$$
\left.\widetilde{v}\right|_{\Gamma_{0}}=0,\left.\quad \widetilde{v}\right|_{\Gamma_{j}}=c_{j}=-4 \operatorname{Im} \iint_{\Omega}\left(A+\frac{\bar{w}}{w} B\right) \sum_{k=1}^{N} c_{j k} \frac{\partial e_{k}}{\partial z} d \Omega, \quad j=1, \ldots, m,
$$

such that the function $f:=w \exp (-\widetilde{w}) \in C^{\alpha}\left(\bar{\Omega}_{m}\right)$ is holomorphic in $\Omega_{m}$ :

$$
\frac{\partial f}{\partial \bar{z}}=0
$$

In fact, $\widetilde{w}$ is given explicitly as

$$
\begin{aligned}
& \left.\widetilde{u}(\xi, \eta)=4 \operatorname{Re} \iint_{\Omega_{m}}\left(A+\frac{\bar{w}}{w} B\right)\right)\left(\frac{\partial}{\partial z} \Gamma^{I I}(z, \zeta)\right) d \Omega, \\
& \left.\widetilde{v}(\xi, \eta)=4 \operatorname{Im} \iint_{\Omega_{m}}\left(A+\frac{\bar{w}}{w} B\right)\right)\left(\frac{\partial}{\partial z} \Gamma^{I}(z, \zeta)\right) d \Omega
\end{aligned}
$$

where $\zeta=\xi+i \eta \in \Omega_{m}$ and $\frac{\bar{w}}{w}$ is set zero wherever $w$ vanishes.

The derivative $\frac{\partial}{\partial z}$ is defined by $\frac{\partial}{\partial z}=\frac{1}{2}\left(\frac{\partial}{\partial x}-i \frac{\partial}{\partial y}\right)$.

Theorem 2.2 (S2). Let $f \in C^{\beta}\left(\bar{\Omega}_{m}\right)$ be holomorphic in $\Omega_{m}, 0<\beta<1$. Then there exists $\widetilde{w} \in C^{\alpha}\left(\bar{\Omega}_{m}\right), \alpha<1-2 / q$ given as a solution of the nonlinear integral equations

$$
\begin{aligned}
\widetilde{v}(\xi, \eta)= & 2 \iint_{\Omega_{m}}\left\{-(\operatorname{Re} A+|B| \cos (p-2 \widetilde{v})) \Gamma_{y}^{I}(z, \zeta)\right. \\
& +\left((\operatorname{Im} A+|B| \sin (p-2 \widetilde{v})) \Gamma_{x}^{I}(z, \zeta)\right\} d \Omega(z) \\
\widetilde{u}(\xi, \eta)= & 2 \iint_{\Omega_{m}}\left\{(\operatorname{Re} A+|B| \cos (p-2 \widetilde{v})) \Gamma_{x}^{I I}(z, \zeta)\right. \\
& +\left((\operatorname{Im} A+|B| \sin (p-2 \widetilde{v})) \Gamma_{y}^{I I}(z, \zeta)\right\} d \Omega(z)
\end{aligned}
$$

where

$$
e^{i p}=\frac{B}{|B|} \frac{\bar{f}}{f} \quad \text { for } B \neq 0 \quad \text { and } \quad p=0 \quad \text { if } B=0
$$

such that $w:=f(z) e^{\widetilde{w}}$ is a solution of $(2.1)$ in $\Omega_{m}$. Moreover, for this specific 
solution we have

$$
\left.\widetilde{v}\right|_{\Gamma_{0}}=0
$$

and

$$
\begin{aligned}
\left.\widetilde{v}\right|_{\Gamma_{j}}= & 2 \sum_{k=1}^{m} c_{k j} \iint_{\Omega_{m}}\left\{\left(\operatorname{Re} A+|B| \cos (p-2 \widetilde{v}) e_{k y}\right.\right. \\
& -\left.\left(\operatorname{Im} A+|B| \sin (p-2 \widetilde{v}) e_{k x}\right\} d \Omega\right|_{\Gamma_{j}} \quad \text { where } j=1, \ldots, m .
\end{aligned}
$$

The harmonic functions $e_{j}$ and the modified Green's and Neumann functions $\Gamma^{I}$ and $\Gamma^{I I}$, respectively, are defined in the Appendix A1, Section 4.

The proofs of these two theorems will be presented in the Appendix A2, Section 4. Note that in general, the solution $\widetilde{w}=\widetilde{u}+i \widetilde{v}$ might not be unique for $m \geqslant 1$. However, one of those solutions is uniquely determined as the limit obtained by the continuation method [20] in combination with the Newton-Kantorovič iteration (4.49), Theorem 4.8, at $t=1$, along the path of problems

$$
\begin{aligned}
\frac{\partial \widetilde{w}^{t}}{\partial \bar{z}} & =t\left\{A+|B| e^{i\left(p-2 \widetilde{v}^{t}\right)}\right\} \quad \text { in } \Omega_{m} \\
\left.\widetilde{v}^{t}\right|_{\Gamma_{0}}=0,\left.\quad \widetilde{v}\right|_{\Gamma_{j}} ^{t}= & 2 t \sum_{k=1}^{m} c_{k j} \iint_{\Omega_{m}}\left\{\left(\operatorname{Re} A+|B| \cos \left(p-2 \widetilde{v}^{t}\right) e_{k y}\right.\right. \\
& -\left.\left(\operatorname{Im} A+|B| \sin \left(p-2 \widetilde{v}^{t}\right) e_{k x}\right\} d \Omega\right|_{\Gamma_{j}}, \quad j=1, \ldots, m,
\end{aligned}
$$

where $t \in[0,1]$, since for $t=0$, the solution of $(2.9)$ is unique. Then the mapping $f \mapsto \widetilde{w}^{1}=: \widetilde{w}$ is well defined which then will be used later on.

\section{The associated Riemann-Hilbert problems}

Now let $w \in C^{\alpha}\left(\bar{\Omega}_{m}\right)$ be a solution of the nonlinear Riemann-Hilbert problem (1.1)-(1.3) where $0<\alpha<1-2 / q$. Then we find with Theorem S1 the function $\widetilde{w}=\widetilde{u}+i \widetilde{v} \in C^{\alpha}\left(\bar{\Omega}_{m}\right)$ given by $(2.3),(2.4)$ such that

$$
f:=w e^{-\widetilde{w}} \in C^{\alpha}\left(\bar{\Omega}_{m}\right) \text { is holomorphic in } \Omega_{m} \text {. }
$$

For $\widetilde{u}$ given by $(2.3)$ we find on $\Gamma_{j}$ the estimates

$$
|\widetilde{u}|_{\Gamma_{j}}\left|\leqslant 4 \max _{\zeta \in \Gamma_{j}} \iint_{\Omega_{m}}(|A|+|B|)\right| \frac{\partial}{\partial z} \Gamma^{I I}(z, \zeta) \mid d \Omega(z)=: K_{j}, \quad j=0, \ldots, m
$$

and from (2.4) for

$$
\begin{aligned}
\left.\widetilde{v}\right|_{\Gamma_{j}}=c_{j} & :=\left.\sum_{k=1}^{m} c_{k j} 4 \operatorname{Im} \iint\left(A+\frac{\bar{w}}{w} B\right)\left(\frac{d}{d z} e_{k}(z)\right) d \Omega(z)\right|_{\Gamma_{j}}, \\
\left|c_{j}\right| & \leqslant\left.\sum_{k=1}^{m} c_{k j} 4 \operatorname{Im} \iint(|A|+|B|)\left|\frac{d}{d z} e_{k}(z)\right| d \Omega(z)\right|_{\Gamma_{j}}=: k_{j},
\end{aligned}
$$


$j=1, \ldots, m$ and $\left.\widetilde{v}\right|_{\Gamma_{0}}=0$. Hence, the holomorphic function $f$ satisfies the nonlinear boundary conditions

$$
f(z) \in \widetilde{\gamma}_{\omega, z, j} \quad \text { for } z \in \Gamma_{j}, \quad j=0, \ldots, m
$$

where $\widetilde{\gamma}_{\omega, z, 1}=\left.e^{-\widetilde{u}(\tau)}\right|_{\Gamma_{0}} \gamma_{\omega, z, 0} \quad$ and $\quad \widetilde{\gamma}_{\omega, z, j}=\left.e^{-\widetilde{u}(\tau)}\right|_{\Gamma_{j}} e^{-i c_{j}} \gamma_{\omega, z, j}, \quad j=1, \ldots, m$

According to (3.5) we now introduce the following families of closed curves characterizing the boundary conditions for $f$ :

$$
\begin{aligned}
\widetilde{\gamma}_{\omega, z, 0}=\left\{\widetilde{\gamma}_{\omega, z, 0}=\varrho_{0}(z) \gamma_{\omega, z, 0} \mid e^{-K_{0}} \leqslant \varrho_{0}(z) \leqslant e^{K_{0}},\right. \\
\left.z \in \Gamma_{0} \wedge \varrho_{0} \in C^{\alpha}\left(\Gamma_{0}\right) \wedge\left\|\varrho_{0}\right\|_{C^{\alpha_{1}}} \leqslant C_{1}\right\}, \\
\widetilde{\gamma}_{\omega, z, j}=\left\{\widetilde{\gamma}_{\omega, z, j}=\varrho_{j}(z) e^{-i c_{j}} \gamma_{\omega, z, j} \mid e^{-K_{j}} \leqslant \varrho_{j}(z) \leqslant e^{K_{j}},\right. \\
\left.\left|c_{j}\right| \leqslant k_{j}, z \in \Gamma_{j} \wedge \varrho_{j} \in C^{\alpha_{1}}\left(\Gamma_{j}\right) \wedge\left\|\varrho_{j}\right\|_{C^{\alpha_{1}\left(\Gamma_{j}\right)}} \leqslant C_{1}\right\} .
\end{aligned}
$$

Then the holomorpic function $f$ defined by the solution $w$ given by (3.1) with $\widetilde{w}$ will satisfy

$$
f(z) \in \widetilde{\gamma}_{\omega, z, j} \quad \text { for } z \in \Gamma_{j}, \quad j=0,1, \ldots, m
$$

for the curves $\widetilde{\gamma}_{\omega, z, j} \in \widetilde{\gamma}_{\omega, z, j}$ in those families, together with

$$
\int_{\Gamma_{j}} d \arg f=0 \text { for } j=0, \ldots, m \text {. }
$$

Now we are in the position to formulate the basic assumptions on our boundary conditions.

Basic Assumptions: (see also (BCG) in [9])

(a) The curves of the families $\gamma_{\omega, z, j}$ with $\omega \in \mathbb{R}^{m-1}$ and $z \in \Gamma_{j}$ for every $j=0,1, \ldots, m$ are at least twice continuously differentiable, closed, nonselfintersecting in the complex state plane $\mathbb{C}_{w}$ and will be parametrized as $z=t_{j}\left(s_{j}\right) \in \Gamma_{j}$, where $0 \leqslant s_{j}<2 \pi$. These curves we denote by $\gamma_{\omega, s_{j}, j}$ and $\widetilde{\gamma}_{\omega, s_{j}, j}$, respectively.

(b) Let $W_{1}<W_{2}<\cdots<W_{2 m+1}$ with $W_{m+1}=0$ be given points on the real axis of the state space $\mathbb{C}_{w}$ and let $W_{2 m+2}$ be the point at infinity of its 1 -point compactification. Then the family of curves must satisfy the following topological conditions:

For each $j=0, \ldots, m$ we assume that all curves in $\widetilde{\gamma}_{\omega, s_{j}, j}$ for $z \in \Gamma_{j}$ and $\omega \in \mathbb{R}^{m-1}$ circumvent the closed interval $\left[W_{j+1}, W_{j+m+1}\right]$ in its exterior but do not include any points of $\left[W_{j+m+2}, W_{j+2 m+2} \bmod (2 m+2)\right]$. 
Recalling Conditions (BCG) in [9], these assumptions are also given as

$$
\begin{aligned}
W_{1} \in \operatorname{int} \widetilde{\gamma}_{\omega, s_{0}, 0} \cap \operatorname{ext} \widetilde{\gamma}_{\omega, s_{j}, j} & \text { for all }\left(\omega, s_{0}\right) \text { and }\left(\omega, s_{j}\right), \\
& j=1, \ldots, m ; \\
W_{k} \in \operatorname{int} \widetilde{\gamma}_{\omega, s_{\ell}, \ell} \cap \operatorname{ext} \widetilde{\gamma}_{\omega, s_{j}, j} & \text { for all }\left(\omega, s_{\ell}\right), \quad \ell=0, \ldots, k-1 ; \\
& \text { and } j=k, \ldots, m ; \quad \text { for } k=2, \ldots, m ; \\
W_{m+1} \in \operatorname{int} \widetilde{\gamma}_{\omega, s_{\ell}, \ell} & \text { for all }\left(\omega, s_{\ell}\right), \quad \ell=0, \ldots, m ; \\
W_{m+1+k} \in \operatorname{int} \widetilde{\gamma}_{\omega, s_{\ell}, \ell} \cap \operatorname{ext} \widetilde{\gamma}_{\omega, s_{j}, j} & \text { for all }\left(\omega, s_{\ell}\right), \quad \ell=k, \ldots, m \\
& \text { and }\left(\omega, s_{j}\right), \quad j=0, \ldots, k-1 ; \\
& \text { for } k=1, \ldots, m ; \\
W_{2 m+2} \in \operatorname{ext} \widetilde{\gamma}_{\omega, s_{j}, j} & \text { for all }\left(\omega, s_{j}\right), \quad j=0, \ldots, m .
\end{aligned}
$$

By int $\gamma$ we denote the bounded component of $\mathbb{C}_{w} \backslash \gamma$. Correspondingly, ext $\gamma$ denotes the unbounded component of $\mathbb{C}_{w} \backslash \gamma$. We further introduce the real intervals $\Delta_{\ell}:=\left[W_{\ell}, W_{\ell+1}\right]$ for $\ell=1, \ldots, 2 m+1$ and $\Delta_{0}:=\left[-W_{2 m+2}, W_{1}\right]$, and corresponding segments in $\mathbb{C}_{w}$. In addition, we require

$$
\Delta_{\ell} \cap \widetilde{\gamma}_{\omega, s_{j}, j}=\emptyset \quad \text { and } \quad \Delta_{m+1+\ell} \cap \widetilde{\gamma}_{\omega, s_{j}, j}=\emptyset \quad \text { for all }\left(\omega, s_{j}\right) \in \mathbb{R} \times[0,2 \pi) \text {, }
$$

where $\ell \neq j$ and $j, \ell=0,1, \ldots, m$.

(c) The curves $\widetilde{\gamma}_{\omega, s_{j}, j}$ lie for every $\left(\omega, s_{j}\right)$ in a closed ring-shaped strip $\Pi_{\omega, j}:=$ $\bigcup_{s_{j}} \widetilde{\gamma}_{\omega, s_{j}, j}$ where $j=0,1, \ldots, m$, respectively, and each of the strips $\Pi_{\omega, j}$, depends continuously on $\omega \in \mathbb{R}^{m-1}$ and is homeomorphic to a circular ring. Moreover, we will assume that for every fixed $\omega$ the strips $\Pi_{\omega, j}$ also satisfy the conditions (b). This means that every simple, closed smooth curve in one of the strips satisfies the conditions (b) correspondingly.

To the point $w \in \mathbb{C}_{w}$ we assign the index $\vec{\alpha}=\left(\alpha_{0}, \alpha_{1}, \ldots, \alpha_{m}\right)$ where $\alpha_{j} \in$ $\{0,1\}$ with $\alpha_{j}=0$ if the point $w \in$ ext $\widetilde{\gamma}_{\omega, s_{j}, j}$ and $\alpha_{j}=1$ if the point $w \in$ int $\widetilde{\gamma}_{\omega, s_{j}, j}$ for $j=0, \ldots, m$. Correspondingly, for given $j$ and $\omega \in \mathbb{R}^{m-1}$, we define the indices for the strips $\Pi_{\omega, j}$ given as follows:

$$
w \in \operatorname{ext} \Pi_{\omega, j}:=\bigcap\left\{\operatorname{ext} \widetilde{\gamma}_{\omega, s_{j}, j} \mid 0 \leqslant s_{j} \leqslant 2 \pi\right\}
$$

or

$$
w \in \operatorname{int} \Pi_{\omega, j}:=\bigcap\left\{\operatorname{int} \widetilde{\gamma}_{\omega, s_{j}, j} \mid 0 \leqslant s_{j} \leqslant 2 \pi\right\}
$$

respectively. We say that a domain $\mathfrak{G}$ of the complex $\mathbb{C}_{w}$-plane has the index $\vec{\alpha}$ if all points in $\mathfrak{G}$ have the index $\vec{\alpha}$. In order to characterize the possible geometric situations we now require for the binary vector function $\vec{\alpha}$ in the $\mathbb{C}_{w}$-plane the following properties.

(d) For $\omega=\left(\omega_{0}, \ldots, \omega_{m-2}\right)$ with $|\omega|<1$, we assume rank $\left(\frac{\partial F_{j}}{\partial \omega_{\ell}}\right)_{\ell=0, \ldots, m-2}^{j=0, \ldots, m}=$ $m-1$, and that the domain $\mathbb{C}_{w} \backslash\left(\bigcup_{j=0}^{m} \widetilde{\gamma}_{\omega, s_{j}, j}\right)$ in the $\mathbb{C}_{w}$-plane consists of $2 m+1$ components which have the indices $\left\{\vec{\alpha}_{1}, \ldots, \vec{\alpha}_{2 m+1}\right\}$. 
(e) For $\omega$ with $|\omega|>2$, we assume that the curves $\widetilde{\gamma}_{\omega, s_{j}, j}$ ) do not depend on $\omega$ anymore and that the domain $\mathbb{C}_{w} \backslash\left(\bigcup_{j=0}^{m} \widetilde{\gamma}_{\omega, s_{j}, j}\right)$ consists of components having the indices $\left\{\vec{\beta}_{1}, \ldots, \vec{\beta}_{2 m+1}\right\} \neq\left\{\vec{\alpha}_{1}, \ldots, \vec{\alpha}_{2 m+1}\right\}$.

Note, then for $|\omega|>2$ no liftable (see Def. 3.1) holomorphic solution can exist due to the open mapping property for holomorphic functions.

For the three-fold connected case $m=2$, the authors give an explicit example for these conditions in [9].

Definition 3.1. The solution $(f(z), \omega)$ with $\omega \in \mathbb{R}^{m-1}$ of the holomorphic Riemann-Hilbert problem in $\Omega_{m}$ subject to the nonlinear boundary conditions (3.5) is called "liftable" if

$$
\begin{gathered}
f\left(\Omega_{m}\right) \subset \bigcup\left\{\operatorname{int} \widetilde{\gamma}_{\omega, s_{j}, j}|\omega \in \mathbb{R}, \quad| \omega \mid<1, \quad 0 \leqslant s_{j} \leqslant 2 \pi \text { andj }=0, \ldots, m\right\}, \\
f(z): \Omega_{m} \rightarrow \mathbb{S}^{2} \backslash\left\{W_{1}, \ldots W_{2 m+2}\right\} \text { is homotopic to a constant solution, }
\end{gathered}
$$

where $\mathbb{S}^{2}$ denotes the standard Riemannian sphere and $W_{j}$ are the corresponding points on $\mathbb{S}^{2}$.

The conditions (a)-(e) become clearly visible on the Riemann sphere $\mathbb{S}^{2}$, if the assumptions are considered on $\mathbb{S}^{2}$ with the images of the intervals and of $\widetilde{\gamma}_{\omega, s_{j}, j}$ for $z \in \Gamma_{j}, \omega \in \mathbb{R}^{m-1}, j=0, \ldots, m$.

The main result of our paper is now formulated in the following theorem.

Theorem 3.1. For $q>4$ and under the Basic Assumptions (a)-(e), the nonlinear Riemann-Hilbert problem (1.1)-(1.3) for the Vekua system has at least two different solutions $\left(w_{+}, \omega_{+}\right)$and $\left(w_{-}, \omega_{-}\right) \in C^{\beta}\left(\bar{\Omega}_{m}\right) \times \mathbb{R}^{m-1}$ and $0<\beta<1-\frac{4}{q}$.

\section{A priori estimates}

As we have seen in Theorem S1, if $w \in C^{\alpha}\left(\bar{\Omega}_{m}\right)$ is a solution of (1.1)-(1.3) then we find a holomorphic function $f \in C^{\alpha}\left(\bar{\Omega}_{m}\right), \frac{1}{2}<\alpha<1-\frac{2}{q}$ and functions $\varrho_{0}$ on $\Gamma_{0}, \ldots, \varrho_{m} \in \Gamma_{m},\left\{\varrho_{0}, \varrho_{1}, \ldots, \varrho_{m} ; c_{1}, \ldots, c_{m}\right\} \in \boldsymbol{\Sigma}_{\alpha}$ where

$$
\begin{aligned}
\boldsymbol{\Sigma}_{\alpha}:=\left\{\left\{\varrho_{0}, \varrho_{1}, \ldots, \varrho_{m} ;\right.\right. & \left.c_{1}, \ldots, c_{m}\right\} \\
\in & \prod_{j=0}^{m} C^{\alpha}\left(\Gamma_{j}\right) \times \mathbb{R}^{m} \mid e^{-K_{j}} \leqslant \varrho_{j} \leqslant e^{K_{j}} \wedge c_{0}=1 \\
& \left.\wedge\left|c_{j}\right| \leqslant k_{j}, \quad\left\|\varrho_{j}\right\|_{C^{\alpha}} \leqslant C_{1}, \quad j=0, \ldots, m\right\} .
\end{aligned}
$$

Theorem 4.1. Let $f$ be the holomorphic solution of the nonlinear RiemannHilbert problem

$$
\frac{\partial f}{\partial \bar{z}}=0 \quad \text { in } \Omega_{m}
$$


and

$$
f(z) \in \varrho_{j}(z) e^{-i c_{j}} \gamma_{\omega, z, j} \quad \text { for } z \in \Gamma_{j}, \quad j=0, \ldots, m
$$

where $c_{0}=0$ and $(\varrho, \mathfrak{c}):=\left\{\varrho_{0}, \ldots, \varrho_{m} ; c_{1}, \ldots, c_{m}\right\} \in \boldsymbol{\Sigma}_{\alpha_{0}}$ with $\frac{1}{2}<\alpha_{0}<1$. Then there exists a constant $C_{0}=C_{0}\left(\alpha_{0}\right)$ such that

$$
\|f\|_{W_{p}^{1-1 / p}(\Gamma)} \leqslant C_{0} \quad \text { and } \quad\|f\|_{C^{\beta}(\Gamma)} \leqslant C_{0}
$$

with

$$
2<p<\left(1-\alpha_{0}\right)^{-1}, \quad 0<\beta<2 \alpha_{0}-1
$$

and

$$
\|f\|_{W_{p}^{1-1 / p}(\Gamma)}:=\|f\|_{L^{p}(\Gamma)}+\left\{\int_{\Gamma} \int_{\Gamma} \frac{|f(t)-f(s)|^{p}}{|t-s|^{p}} d t d s\right\}^{\frac{1}{p}}
$$

The proof will be given in Appendix A3.

Now, for every choice of $\left\{\varrho_{0}, \ldots, \varrho_{m} ; c_{1}, \ldots, c_{m}\right\} \in \boldsymbol{\Sigma}_{\alpha_{0}}$ we have to solve a nonlinear Riemann-Hilbert problem (4.2), (4.3). These problems have been investigated in [9] where we have shown that there exist at least two different solutions $\left(f_{+}, \omega_{+}\right)$and $\left(f_{-}, \omega_{-}\right)$by lifting the problem to the universal covering on the unit disk based on the conformal mapping of two Riemann sheets of the slit complex $\mathbb{C}_{w}$ plane with the real slits

$$
\left[W_{1}, W_{2}\right],\left[W_{3}, W_{4}\right], \ldots,\left[W_{2 m+1}, W_{2 m+2}\right]
$$

to circular $(m+1)$-polygons and their reflections on the unit disc.

For every chosen $(\varrho, \mathfrak{c})=\left\{\varrho_{0}, \ldots, \varrho_{m} ; c_{1}, \ldots, c_{m}\right\} \in \boldsymbol{\Sigma}_{\alpha}$ we obtain at least two different solutions which define two set valued mappings

$$
\begin{aligned}
& \boldsymbol{\Sigma}_{\alpha_{0}} \ni(\varrho, \mathfrak{c}) \mapsto f_{+}=: \mathfrak{A}_{+}(\varrho, \mathfrak{c}): \boldsymbol{\Sigma}_{\alpha_{0}} \mapsto C^{\beta}\left(\bar{\Omega}_{m}\right) \\
& \boldsymbol{\Sigma}_{\alpha_{0}} \ni(\varrho, \mathfrak{c}) \rightarrow f_{-}=: \mathfrak{A}_{-}(\varrho, \mathfrak{c}): \boldsymbol{\Sigma}_{\alpha_{0}} \rightarrow C^{\beta}\left(\bar{\Omega}_{m}\right)
\end{aligned}
$$

With the holomorphic function $f_{+}$or $f_{-}$, respectively, we now apply Theorem 2.2 and obtain to $f_{+}$generalized analytic functions $w_{+}\left(f_{+}\right)$, functions $\widetilde{w}_{+}$ from $(2.5),(2.6)$ and $\left(\varrho_{+}, \mathfrak{c}_{+}\right)$from $(3.5),(3.6)$. Thus we can define the set valued mapping

$$
\begin{aligned}
& \mathfrak{B}_{+}:(\varrho, \mathfrak{c}) \mapsto f_{+} \mapsto \widetilde{w}_{+}\left(\varrho_{+}, \mathfrak{c}_{+}\right), \text {i.e. } \\
& \mathfrak{B}_{+}: \boldsymbol{\Sigma}_{\alpha_{0}} \rightarrow \boldsymbol{\Sigma}_{\alpha_{2}} \Subset \boldsymbol{\Sigma}_{\alpha_{0}}
\end{aligned}
$$

where $\alpha_{2}>\alpha_{0}$.

Correspondingly, we also define

$$
\begin{aligned}
& \mathfrak{B}_{-}:(\varrho, \mathfrak{c}) \mapsto f_{-} \mapsto \widetilde{w}_{-} \mapsto\left(\varrho_{-}, \mathfrak{c}_{-}\right), \text {i.e. } \\
& \mathfrak{B}_{-}: \Sigma_{\alpha_{0}} \rightarrow \Sigma_{\alpha_{2}} \Subset \Sigma_{\alpha_{0}} .
\end{aligned}
$$


Theorem 4.2. Each of the mappings $\mathfrak{B}_{+}$and $\mathfrak{B}_{-}$possesses at least one fixed point $\left(\varrho_{+}^{0}, \mathfrak{c}_{+}^{0}\right) \in \mathfrak{B}_{+}\left(\varrho_{+}^{0}, \mathfrak{c}_{+}^{0}\right) \in \boldsymbol{\Sigma}_{\alpha_{0}}$ and at least one fixed point $\left(\varrho_{-}, \mathfrak{c}_{-}\right) \in \mathfrak{B}_{-}\left(\varrho_{-}^{0}, \mathfrak{c}_{-}^{0}\right) \in$ $\Sigma_{\alpha_{0}}$ where $\frac{1}{2}<\alpha_{1}<\alpha_{0}<\alpha_{2}<1-2 / q$.

For the proof we show the following Lemma.

Lemma 4.3. For any given $(\varrho, c) \in \boldsymbol{\Sigma}_{\alpha_{0}}$ there exist two holomorphic functions $f_{+}$with $\omega_{+} \in \mathbb{R}^{m-1}$ and $f_{-}$with $\omega_{-} \in \mathbb{R}^{m-1}$, which are solutions of the nonlinear Riemann-Hilbert problem

$$
\frac{\partial f}{\partial \bar{z}}=0 \quad \text { in } \Omega_{m}
$$

and

$$
f(z) \in \widetilde{\gamma}_{\omega, z, j} \quad \text { for } z \in \Gamma_{j}, \quad j=0,1, \ldots, m .
$$

Proof of Lemma 4.3. Let us now consider a whole family of nonlinear RiemannHilbert problems $(4.9),(4.10)$ where we associate with any chosen $(\varrho, \mathfrak{c}) \in \boldsymbol{\Sigma}_{\alpha_{0}}$ the family

$$
\begin{array}{ll}
\varrho_{j}(z ; \tau):=1+\left(\varrho_{j}(z)-1\right) \tau, \quad c_{j}(\tau):=\tau c_{j} \\
\quad \text { for } 0 \leqslant \tau \leqslant 1, \quad z \in \Gamma_{j}, \quad j=0, \ldots, m ;
\end{array}
$$

which defines a corresponding family of boundary conditions

$$
\begin{aligned}
& f(z) \in \widetilde{\gamma}_{\omega, z, j, \tau}=\left\{\varrho_{j}(z ; \tau) e^{-i c_{j}} \gamma_{\omega, z, j}\right\} \\
& \text { for } 0 \leqslant \tau \leqslant 1, \quad z \in \Gamma_{j}, \quad j=0, \ldots, m
\end{aligned}
$$

that depends continuously on $\tau$. For each $\tau \in[0,1],(4.11),(4.12)$ defines a nonlinear Riemann-Hilbert problem for holomorphic functions.

However, except for $\tau=0$ (when $\varrho_{j}(z ; 0)=1$ for $j=0, \ldots, m$ ), we cannot apply the arguments as in our paper [9] based on the degree theory for quasiruled Fredholm maps since there we need smooth dependence of the curves

$$
\widetilde{\gamma}_{\omega, t_{j}(s), j}=\varrho_{j}\left(t_{j}(s) ; \tau\right) e^{-i c_{j}(\tau)} \gamma_{\omega, t_{j}(s), j}
$$

on the parameter $s \in \mathbb{R}$, whereas for $0<\tau \leqslant 1$, we only have Hölder continuity of $\varrho_{j}\left(t_{j}(s) ; \tau\right)$ available.

For every $\omega_{j}$ and $s$ fixed, we parameterize the smooth, simply closed curve $\gamma_{\omega, t_{j}(s), j}$ in the complex state plane by the parameter $\sigma \in I$ as the arc length on this regular curve divided by the total length of $\gamma_{\omega, t_{j}(s), j}$. Varying now $s$, the curve varies and varying both parameters, $s$ and $\sigma$ we obtain a regular, doubly periodic $C^{2}$-manifold, the restriction manifold (see [17, Sec. 1.8]),

$$
\mathcal{M}_{j}(\tau, \omega): X=U_{j}(\tau, \omega ; s, \sigma), \quad Y=V_{j}(\tau, \omega ; s, \sigma) \quad \text { for }(s, \sigma) \in I \times I
$$

for each $j=0, \ldots, m$. Since for fixed $s, \tau, j, \omega,(4.14)$ describes a simple, closed plane curve $\gamma_{\omega, t_{j},(s), j}$ and $U_{j}, V_{j}$ are $2 \pi$-periodic in $s$, the manifolds $\mathcal{M}_{j}(\tau, \omega)$ can 
be embedded into the ambient space $\mathbb{R}^{3}$ forming $m+1$ tori for every fixed $\tau \in[0,1]$ and $\omega \in \mathbb{R}^{m-1}$; e.g. by the parametric representations

$$
\begin{aligned}
\mathcal{M}_{j}(\tau, \omega): x_{1} & =\left(R+U_{j}(\tau, \omega ; s, \sigma)\right) \cos s, \quad x_{2}=\left(R+U_{j}(\tau, \omega ; s, \sigma)\right) \sin s, \\
x_{3} & =V_{j}(\tau, \omega ; s, \sigma),
\end{aligned}
$$

where $R>0$ is chosen large enough to avoid selfintersecting.

Hence, if $f(z)$ is a holomorphic solution in $\Omega_{m}$ satisfying (4.10), then on each of the restriction manifolds we find the point $\left(s, \sigma_{j}(s)\right)$ such that

$$
\begin{aligned}
& f\left(t_{j}(s)\right)=U_{j}\left(\tau, \omega ; s, \sigma_{j}(s)\right)+i V_{j}\left(\tau, \omega ; s, \sigma_{j}(s)\right) \\
& \text { for } s \in I, \quad j=0, \ldots, m .
\end{aligned}
$$

Moreover, if $f\left(t_{j}(\bullet)\right) \in C^{\beta}(I)$ and, for $\omega$ and $\tau$ fixed, $\varrho_{j}\left(t_{j}(\bullet) ; \tau\right) \in C^{\beta}(I)$ then $\sigma_{j} \in C^{\beta}(I)$ as well (see Lemma 1.8.2 in [17]). Now we associate with (4.15), (4.9) and (4.10) a nonlinear operator equation for the functions $\sigma_{0}, \ldots, \sigma_{m}$, based on an appropriate representation of $f(z)=u+i v$.

In order to express $v$ on the boundary component $\Gamma_{j}$, we employ the modified Neumann function $\Gamma^{I I}$ and Green's theorem to find the representation

$$
v(\xi, \eta)=\int_{\Gamma} u d \Gamma^{I I}+\int_{\Gamma} v \theta d s \quad \text { for } \zeta=\xi+i \eta \in \Omega_{m}
$$

which holds up to the boundary $\Gamma$. In particular, for $\zeta \in \Gamma_{j}$ we have

$$
\int_{\Gamma} u d \Gamma^{I I}(\zeta)=-\left.\mathcal{H}_{j} u\right|_{\Gamma_{j}}+\int_{\Gamma} u K_{j}(\zeta, z(s)) d s,
$$

where

$$
\mathcal{H}_{j} u_{j}=\frac{1}{\pi} \int_{\Gamma_{j}} u_{j}(s) \frac{d}{d s} \log |\zeta-z(s)| d s
$$

is Hilbert's singular integral operator, and where the integral is defined as Cauchy's principal value. The functions $K_{j}$ are in $C^{2}(\Gamma \times \Gamma)$ (at least). Since each of the closed curves $\Gamma_{j}$ is 1 -periodically parameterized, and on $\Gamma_{j}$ the traces of functions are 1 -periodic, we replace $\mathcal{H}_{j}$ by the Hilbert transform

$$
\mathcal{H} u(t)=\int_{-\frac{1}{2}}^{\frac{1}{2}} u(s) \cot (\pi(s-t)) d s,
$$

and the difference of operators $\mathcal{H}_{j}-\mathcal{H}_{0}$ then has a smooth integral operator kernel (at least in $C^{2}(\Gamma \times \Gamma)$ as well). Hence, with $u_{j}=\left.u\right|_{\Gamma_{j}}$,

$$
\left.v\right|_{\Gamma_{j}}=\mathcal{H} u_{j}+\left.T u\right|_{\Gamma_{j}}+\int_{\Gamma_{0}} v \theta d s
$$


where $T: L_{p}(\Gamma) \rightarrow C^{2}(\Gamma)$ is a continuous linear mapping. Hence, solving the nonlinear Riemann-Hilbert problem, (4.9) and (4.10), equation (4.15) with holomorphic $f$ is equivalent to finding the functions $\sigma_{j} \in C^{\beta}(I)$ and $\omega \in \mathbb{R}^{m-1}$ as the solutions of the nonlinear equations

$$
\begin{gathered}
V_{j}\left(\tau, \omega ; s, \sigma_{j}(s)\right)-\int_{-\frac{1}{2}}^{\frac{1}{2}} \cot \left(\pi\left(s^{\prime}-s\right)\right) U_{j}\left(\tau, \omega ; s^{\prime}, \sigma_{j}\left(s^{\prime}\right)\right) d s^{\prime}-\left.T U\right|_{\Gamma_{j}} \\
\quad-\int_{\Gamma_{0}} V_{0}\left(\tau, \omega ; s^{\prime}, \sigma_{0}(s)\right) \theta\left(s^{\prime}\right) d s^{\prime}=0 \quad \text { for } s \in I, \quad j=0, \ldots, m,
\end{gathered}
$$

together with

$$
\int_{\Gamma_{j}} V_{j}\left(\tau, \omega ; s^{\prime} ; \sigma_{j}\left(s^{\prime}\right)\right) \frac{\partial e_{j}}{\partial n} d s^{\prime}=0 \quad \text { for } j=1, \ldots, m,
$$

where $U=\left(U_{0}, \ldots, U_{m}\right)$.

For $\tau=0$, these equations are equivalent to finding the holomorphic solution of (4.9) and (4.10) for the case $\varrho_{j} \equiv 1$ and $c_{j} \equiv 0$. This case was considered in [9] where we obtained at least two topologically different holomorphic solutions $f_{+}^{0}(z)$ and $f_{-}^{0}(z)$ with $f_{+}^{0}, f_{-}^{0} \in C^{\beta}\left(\bar{\Omega}_{m}\right)$.

Let us return to the equation (4.18) written in short as

$$
A_{\tau}(\omega ; \boldsymbol{\sigma})=0
$$

for $\boldsymbol{\sigma}=\left(\sigma_{0}, \ldots, \sigma_{m}\right) \in C^{\beta}(I)$ and $\omega \in \mathbb{R}^{m-1}$. If we take the linearization with the Frechét derivative about a given solution $(\boldsymbol{\sigma}, \omega)$, then it becomes a system of linear singular integral operators together with a linear mapping in $\mathbb{R}^{m-1}$,

$$
\begin{aligned}
& \left.\left(A_{\tau}^{\prime}(\omega, \boldsymbol{\sigma})(\widetilde{\omega}, \widetilde{\boldsymbol{\sigma}})\right)\right|_{\Gamma_{j}} \\
& =\left\{\begin{array}{l}
\left(\left(\frac{\partial}{\partial \sigma_{j}} V_{j}\right)\left(\tau, \omega ; s, \sigma_{j}(s)\right)\right) \widetilde{\sigma}_{j}(s)-\left(\left.\mathcal{H}\right|_{\Gamma_{j}}\left(\frac{\partial}{\partial \sigma_{j}} U_{j}\right) \widetilde{\sigma}_{j}\right)(s) \\
-\sum_{k=0}^{m}\left(T \frac{\partial U}{\partial \sigma}\right) \widetilde{\sigma}_{k}-\delta_{0 k} \int \frac{\partial V_{0}}{\partial \sigma_{0}} \widetilde{\sigma}_{0}\left(s^{\prime}\right) \sigma\left(s^{\prime}\right) d s^{\prime} ; \\
\sum_{k=1}^{m} \int_{\Gamma_{j}} \frac{\partial V_{j}}{\partial n} d s \widetilde{\omega}_{k}
\end{array}\right.
\end{aligned}
$$

of Fredholm index 0 because of (3.8), operating on $\widetilde{\boldsymbol{\sigma}}$ and $\widetilde{\omega}$. Note that the coefficients are in $C^{\beta}(I)$, and that the last two terms define linear compact operators. Moreover, the right inverse $B_{\tau}(\omega, \boldsymbol{\sigma})$ to $A_{\tau}^{\prime}(\omega, \boldsymbol{\sigma})$ exists for every $\tau \in[0,1]$ and $\omega \in \mathbb{R}^{m-1}$ as will be shown in Appendix A4. The dependence on $\tau$ is uniformly continuous and defines a homotopic path of operators $A_{\tau}$ connecting the case $\tau=0$ with $\tau=1$ on which we can use the a priori estimate (4.4) that guarantees the 
uniform boundedness of $B_{\tau}(\omega, \boldsymbol{\sigma})$ as well. Hence, we can employ the NewtonKantorovič method in [8, Appendix], (Appendix A4, Theorems 4.7 and 4.8) which implies that the set $\mathcal{T}:=\left\{\tau \in[0,1]\right.$ where (4.20) has a solution $\sigma \in C^{\beta}(\Gamma)$, $\left.\omega \in \mathbb{R}^{m-1}\right\}$ is open, and, because of the continuity of $A_{\tau}$, is closed in [0,1] as well. Hence, $\mathcal{T}=[0,1]$. Since for $\tau=0$, we have a solution $f_{-}^{0}(z)$, we find also a solution $f_{-}(z)$ for $\tau=1$, that is of (4.2) and (4.3) which is uniquely associated with $f_{-}^{0}(z)$. If we start with $f_{+}^{0}(z)$, we find a second solution $f_{+}(z)$ (see $[9$, Theorem 7.2]).

In view of Theorem 4.1, we then have $f_{+}, f_{-} \in C^{\beta}\left(\bar{\Omega}_{m}\right)$ with $0<\beta<2 \alpha_{0}-1$.

Note that Lemma 4.3 implies the existence of the two mappings $\mathfrak{A}_{+}$and $\mathfrak{A}_{-}$ in (4.6), respectively (4.5).

Proof of Theorem 4.2. The mapping

$$
\begin{aligned}
& \mathfrak{B}_{+}:(\varrho, \mathfrak{c}) \mapsto f_{+}:=\mathfrak{A}_{+}(\boldsymbol{\varrho}, \mathfrak{c}) \stackrel{T h .2 .2}{\longmapsto} \widetilde{w} \stackrel{T h .2 .1}{\longmapsto}(\widetilde{\varrho}, \widetilde{\mathfrak{c}}), \\
& \mathfrak{B}_{+}: \boldsymbol{\Sigma}_{\alpha_{0}} \rightarrow C^{\beta}\left(\bar{\Omega}_{m}\right) \stackrel{\text { Th.2.2 }}{\longrightarrow} C^{\beta}\left(\bar{\Omega}_{m}\right) \rightarrow C^{\beta}\left(\bar{\Omega}_{m}\right) \stackrel{\text { Th.2.1 }}{\longrightarrow} \boldsymbol{\Sigma}_{\alpha_{2}} \hookrightarrow \boldsymbol{\Sigma}_{\alpha_{0}}
\end{aligned}
$$

with $\alpha_{2}>\alpha_{0}$ is compact because of $\boldsymbol{\Sigma}_{\alpha_{2}} \Subset \boldsymbol{\Sigma}_{\alpha_{0}}$; and $\boldsymbol{\Sigma}_{\alpha_{0}}$ is closed and convex in $\prod_{j=0}^{m} C^{\alpha_{0}}\left(\Gamma_{j}\right) \times \mathbb{R}^{m-1}$. Hence, we apply Schauder's fixed point principle to find a fixed point

$$
\left(\varrho_{+}^{0}, \mathfrak{c}_{+}^{0}\right) \in \mathfrak{B}_{+}\left(\varrho_{+}^{0}, \mathfrak{c}_{+}^{0}\right)
$$

of $\mathfrak{B}_{+}$.

In the same manner we find a fixed point to $\mathfrak{B}_{-}$corresponding to $\mathfrak{A}_{-}$as proposed.

\section{Appendix A1. The modified Green and Neumann functions}

Based on the results in [15, Section IV] by Bogdan Bojarski and [18], we now define the modified Green's function

$$
G^{I}(z, \zeta)=\frac{1}{2 \pi} \ln \frac{1}{|z-\zeta|}+g^{I}(z, \zeta)
$$

of the Laplacian for $\Omega_{m}$ with homogeneous Dirichlet conditions, i.e.

$$
\left.G^{I}(z, \zeta)\right|_{\zeta \in \partial \Omega_{m}}=0 \text { and } \quad z \in \Omega_{m} .
$$

Let $e_{k}(z)$ be the harmonic basis defined by the following properties:

$$
\begin{array}{ll}
\Delta e_{k}=0 \quad \text { in } \Omega_{m}, \quad k=1,2, \ldots, m \\
\text { with }\left.\quad e_{k}\right|_{\Gamma_{j}}=\delta_{k, j}, \quad j=0,1,2, \ldots, m .
\end{array}
$$

Then $\oint_{\Gamma_{k}} \frac{\partial}{\partial n} e_{k} d s \neq 0$ and

$$
a_{j k}:=\int_{\partial \Omega_{n}} e_{j} \frac{\partial}{\partial n} e_{k} d s \quad \text { for } j, k \in\{1,2, \ldots, m\}
$$


defines a positive definite symmetric capacitance matrix. Its inverse $c_{j k}$ with

$$
\sum_{k=1}^{m} c_{j k} a_{k \ell}=\delta_{j \ell}, \quad j, \ell=1,2, \ldots, m
$$

is also positive definite.

Next, we define the modified Green's function by

$$
\Gamma^{I}(z, \zeta):=G^{I}(z, \zeta)-\sum_{j, k=1}^{m} c_{j k} e_{j}(z) e_{k}(\zeta)
$$

which now satisfies the boundary conditions

$$
\begin{aligned}
& \left.\Gamma^{I}(z, \zeta)\right|_{\zeta \in \Gamma_{\ell}}=-\sum_{j=1}^{m} c_{j \ell} e_{j}(z) \text { for } \ell=1, \ldots, m, \\
& \left.\Gamma^{I}(z, \zeta)\right|_{\zeta \in \Gamma_{0}}=0 .
\end{aligned}
$$

For any given $F \in C^{\alpha}\left(\bar{\Omega}_{m}\right)$ and $\varphi \in C^{0}\left(\partial \Omega_{m}\right)$, the function

$$
u:=-\int_{\partial \Omega_{m}} \varphi \frac{\partial}{\partial n} \Gamma^{I} d s-\iint_{\Omega_{m}} F \Gamma^{I} d \Omega
$$

then satisfies

$$
\begin{gathered}
\Delta u=F \quad \text { in } \Omega_{N}, \\
\left.u\right|_{\Gamma_{\ell}}=\varphi-\sum_{j, k=1}^{m} c_{j k}\left\{\oint_{\partial \Omega_{m}} \varphi \frac{\partial e_{j}}{\partial n} d s+\iint_{\Omega_{m}} F e_{j} d \Omega\right\} \delta_{k \ell}, \quad \ell=0, \ldots, m,
\end{gathered}
$$

and

$$
\int_{\Gamma_{\ell}} \frac{\partial u}{\partial n} d s=0 .
$$

Hence, $u$ admits a harmonic conjugate.

Let $\Gamma^{I I}(z, \zeta)$ denote the modified harmonic Neumann's function, given by

$$
\Gamma^{I I}(z, \zeta)=\frac{1}{2 \pi} \ln \frac{1}{|z-\zeta|}+g^{I I}(z, \zeta)
$$

satisfying

$$
\left.\frac{\partial \Gamma^{I I}}{\partial n}\right|_{\partial \Omega_{m}}=-\theta(s) \quad \text { with } \int_{\partial \Omega_{m}} \theta \Gamma^{I I} d s=0
$$

and

$$
\left.\theta\right|_{\Gamma_{0}}>0,\left.\quad \theta\right|_{\Gamma_{j}}=0, \quad j=1, \ldots, m, \quad \int_{\partial \Omega_{m}} \theta d s=\int_{\Gamma_{0}} \theta d s=1,
$$

which is uniquely determined. 
In relation with the Cauchy-Riemann operator one has the following well known lemma, which we quote without proof (see [11, Section 9.2]).

Lemma 4.4. Let $c \in C^{\alpha}\left(\bar{\Omega}_{m}\right)$ and $\psi \in C^{\alpha}(\Gamma)$ with $0<\alpha<1$.

(i) Then $w(\zeta)=u(\zeta)+i v(\zeta)$ given by

$$
\begin{aligned}
& u(\zeta)=-\oint_{\Gamma} \psi \frac{\partial \Gamma^{I}}{\partial n} d s+4 \operatorname{Re} \iint_{\Omega_{m}} c \frac{\partial \Gamma^{I}}{\partial z} d \Omega \\
& v(\zeta)=\oint_{\Gamma} \psi d \Gamma^{I I}+\kappa+4 \operatorname{Im} \iint_{\Omega_{m}} c \frac{\partial \Gamma^{I I}}{\partial z} d \Omega
\end{aligned}
$$

belongs to $C^{\alpha}\left(\bar{\Omega}_{m}\right)$ for every $\kappa \in \mathbb{R}$ and satisfies

$$
\begin{gathered}
\frac{\partial w}{\partial \bar{z}} \quad \text { in } \Omega \\
\left.u\right|_{\Gamma_{0}}=\left.\psi\right|_{\Gamma_{0}} \\
\left.u\right|_{\Gamma_{\ell}}=\left.\psi\right|_{\Gamma_{\ell}}+\sum_{k=1}^{m} c_{k \ell}\left\{\oint_{\Gamma_{k}} \psi \frac{\partial e_{k}}{\partial n} d s-4 \operatorname{Re} \iint_{\Omega} c \frac{\partial e_{k}}{\partial z} d \Omega\right\}
\end{gathered}
$$

for $\ell=1, \ldots, m$.

(ii) If

$$
\oint_{\Gamma_{k}} \psi \frac{\partial e_{k}}{\partial n} d s-4 \operatorname{Re} \iint_{\Omega} c \frac{\partial e_{k}}{\partial z} d \Omega=0 \quad \text { for } k=1, \ldots, m
$$

then, for every $\kappa \in \mathbb{R}$, the function $w$ in (4.27) is the solution of (4.28) in $\Omega$ and $\left.\operatorname{Re} w\right|_{\Gamma}=\psi$ on $\Gamma$.

\section{Appendix A2. The similarity principle}

The Theorem 2.1 (S1) of the similarity principle follows with the choice $\psi=0$ and $\kappa=0$ from the following theorem in [18], which goes back to Bers and Nirenberg $[2,3]$.

Theorem 4.5. Let $w \in C^{\beta}\left(\bar{\Omega}_{m}\right)$ with $0<\beta<1$ be a solution of (2.1) in $\Omega_{m}$ with coefficients $A, B \in L_{q}\left(\Omega_{m}\right)$ where $q>4$. Then for every function $\widetilde{w}=\widetilde{u}+i \widetilde{v}$ given for $\zeta=\xi+i \eta \in \Omega_{m}$ by

$$
\begin{aligned}
& \widetilde{u}(\xi, \eta)=-\oint_{\Gamma} \psi d \Gamma^{I I}+\kappa_{0}+4 \operatorname{Re} \iint_{\Omega_{m}} g(x, y) \frac{\partial \Gamma^{I I}}{\partial z}(x, y ; \xi, \eta) d \Omega \\
& \widetilde{v}(\xi, \eta)=-\oint_{\Gamma} \psi \frac{\partial}{\partial n} \Gamma^{I} d s+4 \operatorname{Im} \iint_{\Omega_{m}} g(x, y) \frac{\partial \Gamma^{I}}{\partial z}(x, y ; \xi, \eta) d \Omega
\end{aligned}
$$


with

$$
g:= \begin{cases}A+B \frac{\bar{w}}{w} & \text { where } w \neq 0, \\ 0 & \text { where } w=0,\end{cases}
$$

and any chosen $\psi \in C^{\alpha}(\Gamma)$ with $0<\alpha \leqslant 1-2 / q$ and $\kappa \in \mathbb{R}$, the function

$$
f(z):=w(z) e^{-\widetilde{w}(z)}
$$

will be holomorphic in $\Omega_{m}$ and $f \in C^{\beta^{\prime}}\left(\bar{\Omega}_{m}\right)$, where $\beta^{\prime}=\min \{\beta, \alpha\}$. Moreover, $\widetilde{w} \in C^{\alpha}\left(\bar{\Omega}_{m}\right)$, and on $\Gamma$ we have

$$
\begin{aligned}
& \left.\widetilde{v}\right|_{\Gamma_{\ell}}=\left.\psi\right|_{\Gamma_{\ell}}+\sum_{k=1}^{m} c_{k \ell}\left\{\oint_{\Gamma_{k}} \psi \frac{\partial e_{k}}{\partial n} d s-4 \operatorname{Im} \iint_{\Omega_{m}} g \frac{\partial e_{k}}{\partial z} d \Omega\right\} \quad \text { for } \ell=1, \ldots, m \\
& \left.\widetilde{v}\right|_{\Gamma_{0}}=\left.\psi\right|_{\Gamma_{0}} .
\end{aligned}
$$

If, in addition, $\psi$ is absolutely continuous, one has

$$
\left.\frac{\partial \widetilde{u}}{\partial n}\right|_{\Gamma} d s=\left.d \psi\right|_{\Gamma} \quad \text { and } \quad \kappa_{0}=\oint_{\Gamma_{0}} \widetilde{u} \theta d s
$$

Proof. Set $W:=i \widetilde{w}$. Then (4.30) and (4.31) is equivalent to the relation (4.27) for $W$,

$$
\begin{aligned}
W(\zeta)= & -\oint_{\Gamma} \psi\left(\frac{\partial \Gamma^{I}}{\partial n}-i \frac{\partial \Gamma^{I I}}{\partial s}\right) d s-i \kappa \\
& +2 \iint_{\Omega_{m}}\left\{(i g)\left(\Gamma_{z}^{I}+\Gamma_{z}^{I I}\right)+\overline{(i g)}\left(\Gamma_{z}^{I}-\Gamma_{\bar{z}}^{I I}\right)\right\} d \Omega,
\end{aligned}
$$

which is the equation (1.1.23) in [21] with $a=b=0$ and $c=i g$. Hence,

$$
\frac{\partial W}{\partial \bar{z}}=i g
$$

and for $f$ defined in (4.33), we get with (4.32)

$$
\frac{\partial f}{\partial \bar{z}}=\frac{\partial w}{\partial \bar{z}} e^{-\widetilde{w}}-w e^{-\widetilde{w}} \frac{\partial \widetilde{w}}{\partial \bar{z}}=e^{-\widetilde{w}}\{A w+B \bar{w}-w g\}=0 \quad \text { in } \Omega,
$$

so, $f$ is holomorphic. As for the Hölder continuity of $\widetilde{w}$, since for $w \in C^{\beta}\left(\bar{\Omega}_{m}\right)$, the function $g$ is measurable and in $L_{q}$ for $q>4$, we find from [15] that $\widetilde{w} \in C^{\alpha}\left(\bar{\Omega}_{m}\right)$ with $0<\alpha \leqslant 1-\frac{2}{q}$. The boundary conditions (4.34) are a consequence of the generalized Green functions' properties.

The proof of the existence of $\widetilde{w}$ in Theorem 2.2 is the same as in [11, Chap.9] for a simply connected domain $\Omega$ except for its uniqueness. To this end, we consider 
the mapping

$$
\begin{aligned}
T(v):= & 2 \iint_{\Omega_{m}}\left\{-(\operatorname{Re} A+|B| \cos (p-2 v)) \Gamma_{y}^{I}(z, \zeta)\right. \\
& \left.+(\operatorname{Im} A+|B| \sin (p-2 v)) \Gamma_{x}^{I}(z, \zeta)\right\} d \Omega
\end{aligned}
$$

for continuous functions $v \in C^{0}\left(\bar{\Omega}_{m}\right)$.

Lemma 4.6. The mapping $T(v)$ has a fixed point $\widetilde{v} \in C^{\alpha}\left(\bar{\Omega}_{m}\right), 0<\alpha<1-\frac{2}{q}<1$ : i.e.,

$$
\widetilde{v}=T(\widetilde{v}) .
$$

Proof. Note that for all $v \in C^{0}\left(\bar{\Omega}_{m}\right)$ the functions $((\operatorname{Re} A+|B| \cos (p-2 v))$ and ( $\operatorname{Im} A+|B| \sin (p-2 v)$ ) belong to $L_{q}\left(\Omega_{m}\right)$ and, hence,

$$
\|T(v)\|_{C^{\alpha}\left(\bar{\Omega}_{m}\right)} \leqslant c_{\alpha}
$$

with a constant $c_{\alpha}$ not depending on $v$, and $0<\alpha<1-\frac{2}{q}$ (see [15]). Hence, the convex and compact subset

$$
\mathcal{B}_{\alpha}:=\left\{v \in C^{\alpha}\left(\bar{\Omega}_{m}\right) \mid\|v\|_{C^{\alpha}\left(\bar{\Omega}_{m}\right)} \leqslant c_{\alpha}\right\}
$$

of the Banach space $C^{0}\left(\bar{\Omega}_{m}\right)$ equipped with the maximum norm is mapped continuously by $T$ into $\mathcal{B}_{\alpha}$. Hence, due to Michael's selection theorem for multivalued mappings, we can use Schauder's fixed point theorem for multivalued maps (see [22, Theorem 9.G and Corollary 2.13]), which provides us with a solution $\widetilde{v} \in C^{\alpha}\left(\bar{\Omega}_{m}\right)$, i.e. a solution of Equation (2.5).

In contrast to the case of a simply connected domain, where $\widetilde{v}$ is uniquely determined, here we use Schauder's fixed point theorem for multivalued mappings. But then we specify among those fixed points the one obtained by applying (2.9) for $t \in[0,1]$, the continuation method in combination with the Newton-Kantorovič iteration (4.49) and get a well defined mapping $f \mapsto \widetilde{w}$.

With $\widetilde{v} \in C^{\alpha}\left(\bar{\Omega}_{m}\right)$ we now compute $\widetilde{u} \in C^{\alpha}\left(\bar{\Omega}_{m}\right)$ from (2.6). Then Lemma 4.4 with $W=i \widetilde{w}$ implies that

$$
\frac{\partial \widetilde{w}}{\partial \bar{z}}=A+|B| e^{i(p-2 \widetilde{v})}
$$

and for $w:=f(z) e^{\widetilde{w}}$ we find

$$
\frac{\partial w}{\partial \bar{z}}=f(z) e^{\widetilde{w}} \frac{\partial \widetilde{w}}{\partial \bar{z}}=f(z) e^{\widetilde{w}}\left\{A+|B| e^{i p-2 i \widetilde{v}}\right\}=A w+B \bar{w}
$$

i.e., $w$ satisfies (1.1) in $\Omega_{m}$.

The boundary relations (2.8) follow from (4.29) for $W=i \widetilde{w}$ and (2.5). This completes the proof of Theorem 2.2. 


\section{Appendix A3: Proof of Theorem 4.1}

Following [8], by using conformal mapping, we can assume that for each $t_{0} \in[0,1)$ and $\varepsilon>0$ there exists $\delta(\varepsilon)>0$ such that for $\left|t-t_{0}\right|<\delta(\varepsilon)$ the boundary condition $F_{j}(\omega, z, u, v)=0$ for $z \in \Gamma_{j}$ for $j=0, \ldots, m$ has the form

$$
u=g_{j}(t, \omega, v),
$$

where the function $g_{j}(t, \omega, v)$ is Hölder continuous with respect to $t$, i.e. in $C^{\alpha}$, and differentiable with respect to $v$, satisfying

$$
\left|\frac{\partial g_{j}}{\partial v}\right|=C_{g_{j}} \varepsilon \quad \text { and } \quad \frac{\left|g_{j}\left(t_{1}, \omega, v\right)-g_{j}\left(t_{2}, \omega, v\right)\right|}{\left|t_{1}-t_{2}\right|^{\alpha}} \leqslant C_{0}
$$

for every fixed $\omega$. Now, the remaining part of the proof of the Zygmund estimates follows closely the presentation in [12]. Due to the maximum principle, for any holomorphic $f(z)=u(z)+i v(z)$ satisfying (4.3) we have

$$
\|f\|_{C^{0}(\Gamma)} \leqslant c_{p}
$$

with the constant $c_{p}$ given by the maximum of $\left|\varrho_{j} e^{-i c_{j}} \gamma_{\omega, z, j}\right|$ for $z \in \Gamma_{j}, j=$ $0, \ldots, m$, and $(\varrho, \mathfrak{c}) \in \boldsymbol{\Sigma}_{\alpha}$.

To prove (4.4), we use the localization principle mentioned above, as well as the representation (4.17) of holomorphic functions on multiply-connected domains $\Omega_{m}$. Let $\varphi$ and $\psi$ in $C^{\infty}\left(\Gamma_{j}\right)$ be supported on $\Gamma_{j}$. Then for fixed $\omega$ and all $t_{j}^{(1)}=t_{j}\left(t_{1}\right)$ and $t_{j}^{(2)}=t_{j}\left(t_{2}\right)$ with $t_{j}^{(1)} \neq t_{j}^{(2)}$ we have

$$
\begin{gathered}
\varphi\left(t_{1}\right) \psi\left(t_{2}\right) \frac{u_{j}\left(t_{1}\right)-u_{j}\left(t_{2}\right)}{t_{1}-t_{2}}=\varphi\left(t_{1}\right) \psi\left(t_{2}\right) \frac{g_{j}\left(t_{1}, v\left(t_{1}\right)\right)-g_{j}\left(t_{2}, v\left(t_{1}\right)\right)}{t_{1}-t_{2}} \\
+\varphi\left(t_{1}\right) \psi\left(t_{2}\right) \frac{g_{j}\left(t_{2}, v\left(t_{1}\right)\right)-g_{j}\left(t_{2}, v\left(t_{2}\right)\right)}{v\left(t_{1}\right)-v\left(t_{2}\right)} \cdot \frac{v\left(t_{1}\right)-v\left(t_{2}\right)}{t_{1}-t_{2}} .
\end{gathered}
$$

In view of (4.39), we define the following operators (according to [12]),

$$
\begin{aligned}
P U\left(t_{1}, t_{2}\right) & :=\varphi\left(t_{1}\right) \psi\left(t_{2}\right) U\left(t_{1}, t_{2}\right), \\
Q u\left(t_{1}, t_{2}\right) & :=\frac{u\left(t_{1}\right)-u\left(t_{2}\right)}{t_{1}-t_{2}}, \\
(B(v))\left(t_{1}, t_{2}\right) & :=\frac{g_{j}\left(t_{2}, v\left(t_{1}\right)\right)-g_{j}\left(t_{2}, v\left(t_{2}\right)\right)}{t_{1}-t_{2}}, \\
(A(v))\left(t_{1}, t_{2}\right) & :=\frac{g_{j}\left(t_{1}, v\left(t_{1}\right)\right)-g_{j}\left(t_{2}, v\left(t_{1}\right)\right)}{t_{1}-t_{2}} .
\end{aligned}
$$

Then (4.39) can be written as

$$
P Q u=P A(v)-P B(v) \cdot \frac{v\left(t_{1}\right)-v\left(t_{2}\right)}{t_{1}-t_{2}} .
$$


Since $P$ is defined by multiplication with $C^{\infty}$-functions, $P$ is a bounded linear operator $P: L^{p}(I \times I) \rightarrow L^{p}(I \times I)$ where $I=[0,1]$ is the periodicity interval. By the definition of the $W_{p}^{1-1 / p}(I)$-norm on $I$, the operator $Q$ acts continuously from $W_{p}^{1-1 / p}(I)$ into $L^{p}(I \times I)$. Further, we have from (4.37) the estimates

$$
\left|B(v)\left(t_{1}, t_{2}\right)\right| \leqslant \varepsilon \quad \text { and } \quad\left|A(v)\left(t_{1}, t_{2}\right)\right| \leqslant c_{0}\left|t_{1}-t_{2}\right|^{\alpha_{0}-1} .
$$

So, we obtain for $2<p<\frac{1}{1-\alpha_{0}}$ together with (4.38)

$$
\|P A(v)\|_{L^{p}(I \times I)} \leqslant c_{p}\|A(v)\|_{L^{p}(I \times I)} \leqslant c_{p} c_{1} ;
$$

since then the space $W_{p}^{1-1 / p}(I) \hookrightarrow C^{\beta}(I)$ is continuously imbedded into the Hölder space $C^{\beta}(I)$ for $\beta<1-2 / q$ (see [14, Remark 2, p.71]); finally,

$$
\|P Q u\|_{L^{p}(I \times I)} \leqslant c_{p} c_{1}+\varepsilon c_{g_{j}}\left|\frac{v\left(t_{1}\right)-v\left(t_{2}\right)}{t_{1}-t_{2}}\right|_{L^{p}(I \times I)} .
$$

For the last term in (4.41), we now employ the relation (4.17) and find on $\Gamma_{j}$ :

$$
\left.\frac{v\left(t_{1}\right)-v\left(t_{2}\right)}{t_{1}-t_{2}}\right|_{I}=\frac{1}{t_{1}-t_{2}}\left(\mathcal{H} u_{j}\left(t_{1}\right)-\mathcal{H} u_{j}\left(t_{2}\right)\right)+\frac{1}{t_{1}-t_{2}}\left(\left.T u\right|_{I}\left(t_{1}\right)-\left.T u\right|_{I}\left(t_{2}\right)\right) .
$$

For the first term on the right-hand side with the Hilbert transform we now exploit 1-periodicity and follow [12], by writing just $u$ for $\left.u\right|_{\Gamma_{j}}$ :

$$
\frac{1}{t_{1}-t_{2}}\left(\mathcal{H} u\left(t_{1}\right)-\mathcal{H} u\left(t_{2}\right)\right)=\int_{-\frac{1}{2}}^{\frac{1}{2}} \frac{u\left(s+t_{1}\right)-u\left(s+t_{2}\right)}{\left(s+t_{1}\right)-\left(s+t_{2}\right)} \cot (\pi s) d s=: G U\left(t_{1}, t_{2}\right),
$$

where $G U:=Q \mathcal{H} u$. With $W(s, t):=U(t, s+t)$ we have

$$
G U(t, r+t)=\int_{-\frac{1}{2}}^{\frac{1}{2}} W(r, s+t) \cot (\pi s) d s
$$

and $L^{p}$ continuity of $\mathcal{H}$ implies

$$
\int_{-\frac{1}{2}}^{\frac{1}{2}}|G U(t, r+t)|^{p} d t \leqslant c \int_{-\frac{1}{2}}^{\frac{1}{2}}|W(r, t)|^{p} d t=\int_{0}^{1}|U(t, r+t)|^{p} d t
$$

Hence,

$$
\|G U\|_{L^{p}(I \times I)}^{p} \leqslant\|U\|_{L^{p}(I \times I)}^{p} .
$$

Finally, we localize again,

$$
U=P U+(I-P) U=P Q u+(I-P) Q u,
$$


and $(I-P) Q$ is continuous from $L_{1}(I)$ into $L_{\infty}(I \times I)$ (see [12]) which yields

$$
\begin{aligned}
\|Q V\|_{L_{p}(I \times I)}^{p \leqslant} & c_{1}\|P Q u\|_{L_{p}(I \times I)}^{p}+c_{1}\|(I-P) Q u\|_{L_{p}(I \times I)}^{p} \\
& +c_{1}\|Q T u\|_{L_{p}(I \times I)}^{p}+\left|\int_{\Gamma} v \sigma d s\right|^{p} \\
\leqslant & c_{1}\|P Q u\|_{L_{p}(I \times I)}^{p}+c_{2}
\end{aligned}
$$

where $c_{2}$ depends on $c_{0}$ and on $\varepsilon$, whereas $c_{1}$ is independent of $\varepsilon$.

Now we insert (4.42) into (4.41) and obtain

$$
\|P Q u\|_{L_{p}(I \times I)}^{p} \leqslant \varepsilon c_{g_{j}} c_{1}\|P Q u\|_{L_{p}(I \times I)}^{p}+c_{p} c_{1}+\varepsilon c_{g_{j}} c_{2} .
$$

Finally, by choosing $\varepsilon>0$ small enough to satisfy $\varepsilon c_{g_{j}} c_{1} \leqslant \frac{1}{2}$, we obtain the first estimate in (4.4). Now, if $0<\beta<2 \alpha_{0}-1$ and $2<p<\left(1-\alpha_{0}\right)$, then $\beta<1-2 / p$ and $W_{p}^{1-1 / p}(I)$ is continuously imbedded into $C^{\beta}(I)$ (see [14, (1.3.10), Remark 2 and (2.2.11) on p.71], which yields the second proposition in (4.4). Since $\frac{1}{2}<\alpha_{0}<1-\frac{2}{q}$ (cf. Theorem 4.2) we find $0<\beta<1-\frac{4}{q}$.

\section{Appendix A4. The Newton-Kantorovič method}

From [8] we quote the following results.

Theorem 4.7. Let $\mathcal{A}: X \rightarrow Y$ be a continuous mapping where $X$ and $Y$ are Banach spaces, and let $\mathcal{A}$ be Frechét differentiable with Frechét derivative $\mathcal{A}_{x}^{\prime} \in$ $\mathcal{L}(X, Y)$, which is Lipschitz continuous:

$$
\left\|\mathcal{A}_{x_{0}}^{\prime}-\mathcal{A}_{x}^{\prime}\right\|_{\mathcal{L}(X, Y)} \leqslant \lambda_{2}\left\|x_{0}-x\right\|_{X}
$$

with $\lambda_{2} \geqslant 0$.

Let $\mathcal{A}_{x_{0}}^{\prime}$ possess a bounded right inverse

$$
\mathcal{B}_{x_{0}}: Y \rightarrow \widetilde{X}:=\mathcal{B}_{x_{0}} Y \subset X \quad \text { with } \lambda_{1}:=\left\|\mathcal{B}_{x_{0}}\right\|_{\mathcal{L}(X, Y)}
$$

Then there exists $\varepsilon>0$ such that for every $x$ with $\left\|x-x_{0}\right\|<\varepsilon$ the Frechét derivatives $\mathcal{A}_{x}^{\prime}$ have also bounded right inverses $\mathcal{B}_{x}$ and

$$
\left\|\mathcal{B}_{x}\right\|_{\mathcal{L}(Y, X)} \leqslant \frac{\lambda_{1}}{1-\lambda_{1} \lambda_{2}\left\|x_{0}-x\right\|_{X}} .
$$

Proof. For the restricted mapping

$$
\widetilde{\mathcal{A}}_{x_{0}}^{\prime}:=\left.\mathcal{A}_{x_{0}}^{\prime}\right|_{\tilde{X}}
$$

we have

$$
\widetilde{\mathcal{A}}_{x_{0}}^{\prime} \mathcal{B}_{x_{0}} y=y \quad \text { for all } y \in Y \quad \text { and } \quad \mathcal{B}_{x_{0}} \widetilde{\mathcal{A}}_{x_{0}}^{\prime} x=x \quad \text { for all } x \in \tilde{X}
$$


Since for

$$
\left\|\mathcal{B}_{x_{0}}\left(\mathcal{A}_{x_{0}}^{\prime}-\mathcal{A}_{x}^{\prime}\right)\right\|_{\mathcal{L}(X, X)} \leqslant \lambda_{1} \lambda_{2}\left\|x_{0}-x\right\|_{X}<1
$$

the inverse

$$
\left[I-\mathcal{B}_{x_{0}}\left(\mathcal{A}_{x_{0}}^{\prime}-\mathcal{A}_{x}^{\prime}\right)\right]^{-1}=\sum_{\ell=0}^{\infty}\left(\mathcal{B}_{x_{0}}\left(\mathcal{A}_{x_{0}}^{\prime}-\mathcal{A}_{x}^{\prime}\right)\right)^{\ell}
$$

exists, we find for the restricted operators

$$
\left.\widetilde{\mathcal{A}}_{x}\right|_{\tilde{X}}: \widetilde{X} \rightarrow Y
$$

the inverse $\mathcal{B}_{x}:=\left(\widetilde{\mathcal{A}}_{x}^{\prime}\right)^{-1}$ as

$$
\mathcal{B}_{x}:=\sum_{\ell=0}^{\infty}\left(\mathcal{B}_{x_{0}}\left(\widetilde{\mathcal{A}}_{x_{0}}^{\prime}-\widetilde{\mathcal{A}}_{x}^{\prime}\right)\right)^{\ell} \mathcal{B}_{x_{0}}
$$

which defines a right inverse to $\mathcal{A}_{x}^{\prime}$. So, if $\mathcal{B}_{x_{0}}$ is the whole bundle of right inverses, we obtain with the family of spaces $\widetilde{X}$ also the bundle of right inverses $\mathcal{B}_{x}$. Moreover,

$$
\left\|\mathcal{B}_{x}\right\|_{\mathcal{L}(Y, X)} \leqslant \frac{\lambda_{1}}{1-\lambda_{1} \lambda_{2}\left\|x-x_{0}\right\|}
$$

for $\lambda_{1} \lambda_{2}\left\|x-x_{0}\right\|<1$ and $\varepsilon:=\left(\lambda_{1} \lambda_{2}\right)^{-1}$. If $\left\|x_{0}-x\right\|<\left\{2\left(\lambda_{1}+1\right)\left(\lambda_{2}+1\right)\right\}^{-1}$ then uniformly

$$
\left\|\mathcal{B}_{x}\right\|_{\mathcal{L}(Y, X)}<2 \lambda_{1}
$$

Theorem 4.8. Let $x_{0} \in X$ and $\mathcal{A}$ have the properties as in Theorem 4.7. Then if

$$
y=\mathcal{A}\left(x_{0}\right) \in Y
$$

with

$$
\|y\|_{Y}<\lambda_{3}:=\min \left\{\left(4 \lambda_{1}^{2} \lambda_{2}\right)^{-1},\left(2\left(\lambda_{1}+1\right)\left(\lambda_{2}+1\right)\right)^{-1}\right\},
$$

there exists a solution $x \in X$ of

$$
\mathcal{A}(x)=0
$$

with

$$
\left\|x-x_{0}\right\|_{X}<\left\{2\left(\lambda_{1}+1\right)\left(\lambda_{2}+1\right)\right\}^{-1} .
$$

This solution is uniquely determined by Newton's method:

$$
x_{k+1}:=x_{k}-\mathcal{B}_{x_{k}} f_{k} \quad \text { and } \quad f_{k+1}:=\mathcal{A}\left(x_{k+1}\right),
$$

where $f_{0}:=-y$ and $x=\lim _{k \rightarrow \infty} x_{k}$. Hence, for $y$ satisfying (4.47), the mapping $y \mapsto x$ is well defined and continuous. 
Proof. From (4.49) we obtain

$$
x_{k+1}=x_{k}+h_{k} \quad \text { with } h_{k}=-\mathcal{B}_{x_{k}} f_{k},
$$

and from

$$
\begin{aligned}
\mathcal{A}\left(x_{k}+h_{k}\right) & =\mathcal{A}\left(x_{k}\right)+\int_{0}^{1} \mathcal{A}_{x_{k}+t h_{k}}^{\prime} h_{k} d t=\mathcal{A}\left(x_{k}\right)+\mathcal{A}_{x_{k}}^{\prime} h_{k}+R, \\
R & =\int_{0}^{1}\left(\mathcal{A}_{x+t h_{k}}^{\prime}-\mathcal{A}_{x_{k}}^{\prime}\right) h_{k} d t
\end{aligned}
$$

the relation

$$
f_{k+1}=f_{k}-\mathcal{A}_{x_{k}}^{\prime} \mathcal{B}_{x_{k}} f_{k}+R=R \text {. }
$$

Hence, with (4.43)

$$
\left\|f_{k+1}\right\|=\|R\| \leqslant \lambda_{2} \int_{0}^{1} t\left\|h_{k}\right\|^{2} d t=\frac{1}{2} \lambda_{2}\left\|h_{k}\right\|^{2} .
$$

If we guarantee that $\left\|x_{k}-x_{0}\right\|<\left\{2\left(\lambda_{1}+1\right)\left(\lambda_{2}+1\right)\right\}^{-1}$ then we obtain from (4.46)

$$
\left\|f_{k+1}\right\| \leqslant 2 \lambda_{1}^{2} \lambda_{2}\left\|f_{k}\right\|^{2} \leqslant 2 \lambda_{1}^{2} \lambda_{2} \lambda_{3}\left\|f_{k}\right\|
$$

if $\left\|f_{k}\right\| \leqslant \lambda_{3}$. So, with $q:=2 \lambda_{1}^{2} \lambda_{2} \lambda_{3}$,

$$
\left\|f_{k}\right\| \leqslant q^{k}\left\|f_{0}\right\| \leqslant q^{k} \lambda_{3} .
$$

Hence, with (4.46),

$$
\begin{gathered}
\left\|x_{k+1}-x_{k}\right\|=\left\|h_{k}\right\|=\left\|\mathcal{B}_{x_{k}} f_{k}\right\| \leqslant 2 \lambda_{1}\left\|f_{k}\right\| \leqslant 2 \lambda_{1} \lambda_{3} q^{k}, \\
\left\|x_{k}-x_{0}\right\| \leqslant \frac{2 \lambda_{1} \lambda_{3}}{1-q} .
\end{gathered}
$$

Therefore, we choose $\lambda_{3}>0$ such that

$$
\lambda_{3}=\min \left\{\left(4 \lambda_{1}^{2} \lambda_{2}\right)^{-1},\left\{2\left(\lambda_{1}+1\right)\left(\lambda_{2}+1\right)\right\}^{-1}\right\} .
$$

Then $q=\frac{1}{2}, \lim _{k \rightarrow \infty} x_{k}=x$ exists, and for every $k$ we have $\left\|x_{k}-x_{0}\right\|<\left\{2\left(\lambda_{1}+1\right)\right.$ $\left.\times\left(\lambda_{2}+1\right)\right\}^{-1}$, and $x$ satisfies (4.48).

Now we are in the position to apply the continuation method to (4.20) with the family $A_{\tau}$ of operators with $\tau \in \mathcal{T} \subseteq[0,1]$ and $X:=\mathbb{R}^{m-1} \times C^{\beta}(\Gamma)=Y$. The operators depend continuously on $\tau$ and for every $\tau \in[0,1]$ the Frechét derivative (4.21) exists. For $\tau_{0}=0,(4.20)$ has a solution, and the Frechét derivative possesses a right inverse. Theorems 4.7 and 4.8 show that $\mathcal{T}$ is open since if $A_{\tau_{0}}\left(\omega_{\tau_{0}}, \sigma_{\tau_{0}}\right)=0$ has a solution and $A_{\tau_{0}}^{\prime}\left(\omega_{\tau_{0}}, \boldsymbol{\sigma}_{\tau_{0}}\right)$ has a right inverse, then $A_{\tau_{1}}\left(\omega_{\tau_{1}}, \boldsymbol{\sigma}_{\tau_{1}}\right)=0$ will also admit a solution, and $A_{\tau_{1}}^{\prime}\left(\omega_{\tau_{1}}, \boldsymbol{\sigma}_{\tau_{1}}\right)=0$ has a right inverse if $y=A_{\tau_{1}}\left(\omega_{\tau_{0}}, \boldsymbol{\sigma}_{\tau_{0}}\right)$, which is the case for $0<\left|\tau_{1}-\tau_{0}\right|$ small enough. Because of (4.50), if $\tau_{1} \rightarrow \tau_{0}$, also $\left(\omega_{\tau_{0}}, \boldsymbol{\sigma}_{\tau_{1}}\right) \rightarrow\left(\omega_{\tau_{0}}, \boldsymbol{\sigma}_{\tau_{0}}\right)$ in $X$ since $y \rightarrow 0$. So, $\mathcal{T}$ is also closed. Consequently, $\mathcal{T}=[0,1]$ as proposed in Appendix A4. 
Acknowledgements. The authors thank the referee for his thorough review which helped to improve the original maniscript significantly. The second author acknowledges support by the SFB 404, Multifieldproblems, of the German Research Foundation DFG at the University of Stuttgart.

\section{References}

[1] H. Begehr, C.G. Hsiao, The Hilbert boundary value problem for nonlinear elliptic systems, Proc. Royal Soc. Edinburgh 94A (1983), 97-112.

[2] L. Bers, The Theory of Pseudoanalytic Functions. Lecture Notes, New York Univ., New York, 1953.

[3] L. Bers, L Nirenberg, On a representation theorem for linear elliptic systems with discontinuous coefficients and its applications, Atti di Convegno internationale sulle Equazioni alle derivate parziali, Trieste, 1954, 111-140.

[4] B. Bojarski, T. Iwaniec, Quasiconformal mappings and non-linear elliptic equations in two variables, I. Bull. de L'Academie Polonaise des Siences. Ser. des Sci. math., astr. et phys. XXII No. 5 (1974), 473-478.

[5] B. Bojarski, T. Iwaniec, Quasiconformal mappings and non-linear elliptic equations in two variables, II. Bull. de L'Academie Polonaise des Siences. Ser. des Sci. math., astr. et phys. XXII No. 5 (1974), 479-484.

[6] M. Efendiev, W.L. Wendland, Nonlinear Riemann-Hilbert problems for multiply connected domains, Nonlinear Analysis, Theory, Methods \& Applications 27 (1996), 37-58.

[7] M. Efendiev, W.L. Wendland, Nonlinear Riemann-Hilbert problems for doubly connected domains and closed boundary data, Topological Methods in Nonlinear Analysis. J. of the J. Schauder Center 17 (2001), 111-124.

[8] M. Efendiev, W.L. Wendland, On boundary-layer type solutions of nonlinear Riemann-Hilberts and related geometric structures, Proc. Royal Soc. London A 458 (2002), 539-562.

[9] M.A. Efendiev, W.L. Wendland, Geometrical properties of nonlinear maps and their application; Part II: Nonlinear Riemann-Hilbert problems with closed boundary data for multiply connected domains, J. Math. Anal. Appl. 329 (2007), 425-444.

[10] F.D. Gakhov, Boundary Value Problems, Pergamon Press, New York, 1966. (Russian orig. Fizmatgiz., Moscow 1963.)

[11] W. Haack, W.L. Wendland, Lectures on Partial and Pfaffian Differential Equations, Pergamon Press, Oxford, 1972.

[12] M. Reissig, E. Wegert, Nonlinear boundary value problems for elliptic systems in the plane, Complex Variables 27 (1995), 193-210.

[13] A.I. Šnirelman, The degree of quasi-ruled mapping and a nonlinear Hilbert problem, Math. USSR Sbornik 18 (1974), 373-396. (Russian orig. Mat. Sb. 89,3 (1972), 366-389.)

[14] H. Triebel, Spaces of Besov-Hardy-Sobolev Type, B.G. Teubner, Leipzig, 1978.

[15] I.N. Vekua, Generalized Analytic Functions, Pergamon Press, Oxford, 1962. (Russian orig. Nauka, Moscow, 1959.) 
[16] I.N. Vekua, New Methods for Solving Elliptic Equations, North Holland, Amsterdam, 1968. (Russian orig. Nauka, Moscow, 1948.)

[17] E. Wegert, Nonlinear Boundary Value Problems for Holomorphic Functions and Singular Integral Equations, Akademie Verlag, Berlin, 1992.

[18] W.L. Wendland, On boundary value problems for generalized analytic functions, In: Lecture Notes in Mathematics 280 (N. Everitt, B. Sleeman eds.) Springer-Verlag, Berlin 1972, 190-201.

[19] W.L. Wendland, On a class of semilinear boundary value problems for certain elliptic systems in the plane, In: Complex Analysis and its Applications. Akad. Nauk SSR. To the 70th birthday of Academician I.N. Vekua. Izdat. "Nauka" 1978, 108-119.

[20] W.L. Wendland, On the embedding method for semilinear first order elliptic systems and related finite element methods, In: Continuation Methods (H. Wacker ed.). Academic Press, New York, 1978, 277-336.

[21] W.L. Wendland, Elliptic Systems in the Plane, Pitman, London, 1979.

[22] E. Zeidler, Nonlinear Functional Analysis and its Applications. I Fixed Point Theorems, Springer-Verlag, New York, 1986.

Addresses: M.A. Efendiev: Helmholtz-Center Munich, Institute of Biomathematics and Biometry Ingolstädter Landstraße 1, D-85764 Neuherberg, Germany;

W.L. Wendland: Department of Mathematics, Universiy of Stuttgart, IANS Pfafffenwaldring 57, D-70569 Stuttgart;

E-mail: messoud.efendiyev@gsf.de, wendland@mathematik.uni-stuttgart.de

Received: 27 May 2008; revised: 4 May 2009 